ARTICLE

https://doi.org/10.1038/s41467-018-07781-y

\title{
The propagation of active-passive interfaces in bacterial swarms
}

\author{
Alison E. Patteson ${ }^{1,2}$, Arvind Gopinath ${ }^{3,4} \&$ Paulo E. Arratia ${ }^{1}$
}

Propagating interfaces are ubiquitous in nature, underlying instabilities and pattern formation in biology and material science. Physical principles governing interface growth are well understood in passive settings; however, our understanding of interfaces in active systems is still in its infancy. Here, we study the evolution of an active-passive interface using a model active matter system, bacterial swarms. We use ultra-violet light exposure to create compact domains of passive bacteria within Serratia marcescens swarms, thereby creating interfaces separating motile and immotile cells. Post-exposure, the boundary re-shapes and erodes due to self-emergent collective flows. We demonstrate that the active-passive boundary acts as a diffuse interface with mechanical properties set by the flow. Intriguingly, interfacial velocity couples to local swarm speed and interface curvature, raising the possibility that an active analogue to classic Gibbs-Thomson-Stefan conditions may control this boundary propagation.

\footnotetext{
${ }^{1}$ Department of Mechanical Engineering \& Applied Mechanics, University of Pennsylvania, Philadelphia, PA 19104, USA. ${ }^{2}$ Physics Department, Syracuse University, Syracuse, NY 13244, USA. ${ }^{3}$ Department of Bioengineering, University of California, Merced, CA 95340, USA. ${ }^{4}$ Health Sciences Research Institute, University of California, Merced, CA 95340, USA. Correspondence and requests for materials should be addressed to

A.E.P. (email: aepattes@syr.edu) or to P.E.A. (email: parratia@seas.upenn.edu)
} 
B acteria live and move in an extraordinarily wide range of habitats and can quickly respond to the presence of other cells and physical boundaries in their environment. For instance, bacteria swim independently in fluids, but when transferred to surfaces display a collective behavior known as swarming ${ }^{1,2}$. Swarming occurs in many gram-negative and grampositive species and corresponds to a hyper-flagellated elongated phenotype ${ }^{2,3}$. Swarming cells self-organize into rapid collective motions that allow for quick colonization of new environmental niches ${ }^{1,4-6}$. Swarming is co-regulated with virulence determinants, inversely regulated with sessile biofilm formation, and associated with enhanced antibiotic resistance ${ }^{6-8}$. More broadly, the collective motion of self-propelling (active) particles 9,10 is observed in bacterial infections ${ }^{11}$, embryogenesis ${ }^{12}$, and wound healing ${ }^{13}$ and is an important feature of both prokaryotic ${ }^{14}$ and eukaryotic ${ }^{15}$ systems.

Ecological niches are typically a heterogeneous mix of cells, and internal boundaries can form separating cells of two difference types. Bacterial swarms coexist symbiotically with other microbes -assisting in the transport of fungal spores ${ }^{16}$ and other bacterial species $^{17}$ - or they compete at sharp boundaries ${ }^{18,19}$. Boundaries also emerge in cultures of the same bacteria due to chemotaxis and cell death ${ }^{20,21}$ or the presence of extracellular polymers 22,23 , both of which can induce a swimming-speed dependent phase separation $^{21,23}$. Segregation of active particles is not unique to biological settings, arising in synthetic systems such as phoretic particles ${ }^{24,25}$.

In passive bi-phasic systems ${ }^{26-28}$ such as melting ice-water mixtures and solidifying alloys, properties of internal boundaries (such as interface shape and speed) depend on the surface tension, interfacial energies, and externally imposed flows. In active systems, particle motion can couple to the presence of boundaries which can lead to anomalous properties in mechanical pressure $^{29,30}$ and impact collective flows ${ }^{31,32}$. However, despite the ubiquity of boundaries in living and life-like materials, boundary stability and motion remain largely unexplored in active non-equilibrium matter. Identifying boundary conditions that link boundary structure and active motion will help elucidate a minimal description of actively-driven boundaries.

Here, we focus on the propagation of an interface separating mobile and immobile bacteria in swarming Serratia marcescens. Serratia are a rod-shaped, gram-negative, opportunistic pathogen of the Enterobacteriaceae family ${ }^{3}$. We use high intensity ultraviolet (UV) light to selectively paralyze and passivate cells in large compact domains within the swarm (Methods). The passive domain and active swarm interact at the interphase boundary where self-emergent, vortical flows develop. The interphase is spontaneously reshaped and eroded as passivated bacteria are dislodged from their neighbors and convected by nearby collective flows. Intriguingly, the active-passive boundary behaves as a propagating, diffuse elastic interface with speeds that seems to correlate with local interface curvature and the intensity of the active bacterial flow. Our results raises the possibility that an active analog to classic Gibbs-Thomson-Stefan conditions may control the observed boundary propagation process.

\section{Results}

Generating passive domains in bacterial swarms. Figure 1a shows snapshots of a Serratia marcescens swarm before and after its exposure to UV light. The swarm is grown on an agar substrates (Methods 1) and is pictured moving right to left at a speed of approximately $1 \mu \mathrm{m} / \mathrm{min}$; the colony edge is marked by a clear precursor fluid film (white edge in image). Close examination reveals (Fig. 1b) densely packed rod-shaped cells with local orientational order resembling a nematic liquid crystal. The individual cells ${ }^{3}$ are $1 \mu \mathrm{m}$ in diameter and 5-7 $\mu \mathrm{m}$ in length, and the collective swarm edge is estimated to be approximately a monolayer thick based on previous investigations ${ }^{5}$. In its initial state, the swarm is highly motile and exhibits long-range collective flows (Supplementary Movie 1). We use particle image velocimetry (PIV, Methods 0.2, Supplementary Notes 1 and 2) to exact the bacterial velocity field $\mathbf{v}(\mathbf{r}, t)$ (overlaid in color). We find that the fastest moving cells are approximately 100-400 $\mu \mathrm{m}$ from the leading edge of the colony, where the average local speed is 28 $\mu \mathrm{m} / \mathrm{s}$ (Fig. 1c). The collective flow has a correlation length of approximately $20 \mu \mathrm{m}$ and characteristic time of $0.25 \mathrm{~s}$ (Supplementary Note 2, Supplementary Fig. 1).

When exposed to sufficiently high intensity light (Methods 3, Supplementary Note 1), a portion of the highly motile swarm is quenched and becomes immobile (Fig. 1a, Supplementary Movie 2). We use standard microscope optics to focus the light from a mercury lamp source to the swarm, selectively damaging and blocking cell motility [Methods 3]. This generates domains of passive bacteria, the shape of which can be controlled through an aperture. We focus on two aperture geometries: a half-plane aperture $[\mathrm{H}]$ and an octagonal-shaped $[\mathrm{O}]$ aperture.

Figure 1 illustrates the swarm's response to UV light using the [O] aperture. Bacteria inside the exposed region (highlighted octagon) stop moving and are eventually trapped. This is seen in Supplementary Movie 2: particles that serve as tracers slow down and eventually stop moving as they are trapped amongst the passive bacteria. The slowing down is accompanied by increasing alignment in the exposed region; such alignment may be due to direct excluded volume interactions ${ }^{33}$ or via induced slowly decaying flows. Bacteria outside the exposure remain motile, forming vortices and jets along the boundary of the trapped cells (Supplementary Movie 3).

When the light source is switched off, the active unexposed bacteria collide with the region of passivated bacteria. Initially, the passivated bacteria are trapped in space by their tightly packed neighbors. The incessant motions of the swarm dislodge passivated bacteria from their neighbors and convects them from the boundary toward the active region; the passive bacteria appear absorbed by the swarm, as shown in Supplementary Movies 4-6. Simultaneously, active bacteria move into the passive regions and the interface propagates radially inward albeit asymmetrically. The swarm's collective motion recovers to its pre-exposure state (Fig. 1c).

Propagating interfaces of bacteria have been recently reported $^{34}$; the use of a rotating magnet to impose vortical flows ${ }^{34,35}$ leads to an accumulation of bacteria in suspension, forming dense bacterial droplets that expand in a violent instability when released. In our experiments, we observe a different transport mechanism, erosion of a solid phase of passive particle by an active swarm of bacteria with emergent collective flows. Here, we will present new findings that the presence of soft deformable boundaries impacts the collective motion of swarming flows.

Flow streamlines (Fig. 1d) near the interphase boundary show the rich complex behaviors of individual vortices. We observe vortices convecting in from the bulk, interacting with each other, and lingering at the surface (highlighted brown vortex, for example). Similar features occur when the exposure is made using the $[\mathrm{H}]$ aperture (Supplementary Fig. 2, Supplementary Movie 5); a notable difference for the $[\mathrm{H}]$ aperture is that the absence of imposed corners and the approximate translational invariance along the exposed edge results in active-passive boundary propagating without large scale curvatures.

$\mathrm{Bi}$-phasic passive systems involving boundary erosion such as melting of ice or alloy solidification usually feature a diffuse interfacial region where the two phases mix. The active system here differs from these classical systems in some noteworthy ways. Here, the passive region is continuously eroded and remodeled by self-emergent self-organized flows generated in the 

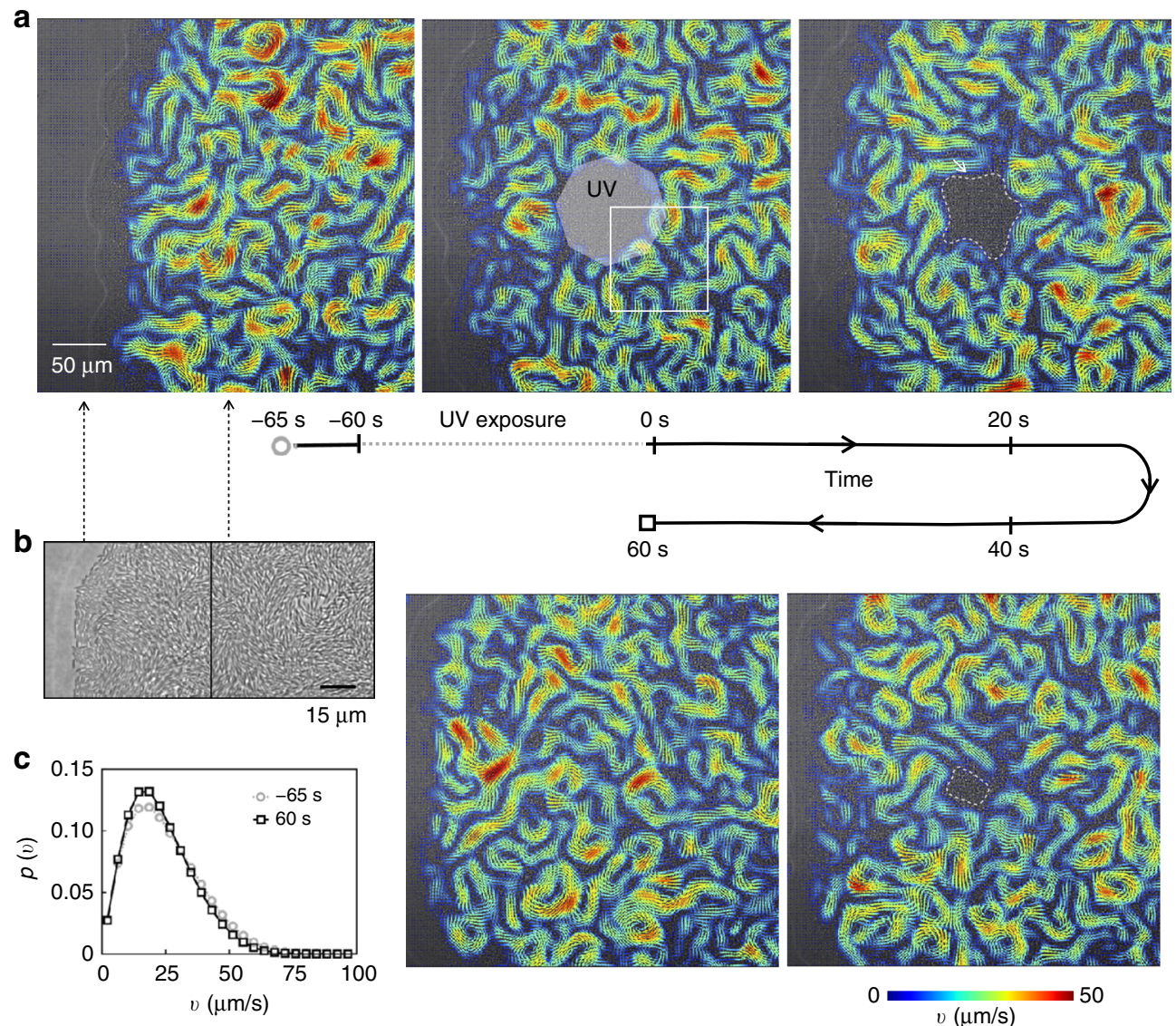

d

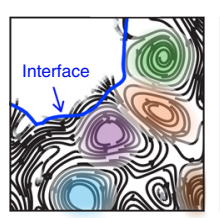

$t=0.0 \mathrm{~s}$

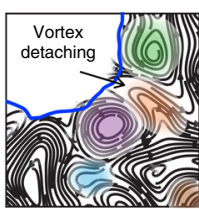

$0.3 \mathrm{~s}$

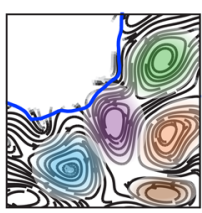

$0.6 \mathrm{~s}$

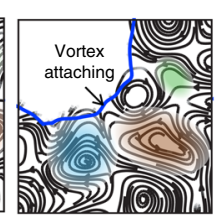

$0.9 \mathrm{~s}$

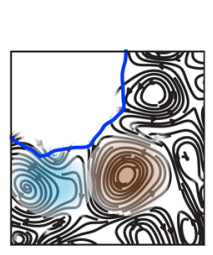

$12 \mathrm{~s}$

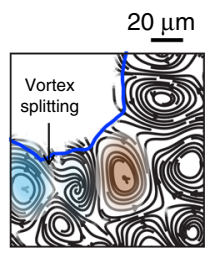

$1.5 \mathrm{~s}$

Fig. 1 Creation and dissolution of an active-passive interphase boundary in a bacterial swarm. a A series of snapshots of the expanding edge of a Serratia marcescens swarm taken over time. The colony was cultured on an agar substrate, and its expanding edge (marked by a precursor fluid film that appears as white curve) is moving from right to left. The swarm shows long-range collective flows, with strong velocity fields (PIV; overlaid color). A large domain of passive, immobile bacteria is created by exposing a region of the swarm to high intensity ultraviolet (UV) light (highlighted octagon). An interphase boundary forms between the passivated and active bacteria. When the light source is switched off ( $t=0 \mathrm{~s}$ ), the active unexposed bacteria deform the interphase boundary and penetrate the passive region. Over time, active bacteria convect immobile bacteria away from the passive domain, causing the boundary to erode and propagate inward. The boundary is manually traced in white for visualization. The swarm dissolves the passive phase in $60 \mathrm{~s}$, with interface speeds greater than that of expanding colony edge. b The swarm edge (close-up) features densely packed cells with local polarity and nematic order. c The swarm's collective motion recovers after dissolution as shown by the probability distribution of bacterial speeds $p(v)$, before and after exposure (data shown for representative experiment, collected from $5 \mathrm{~s}$ interval of PIV data). d A montage of the flow streamlines-from the highlighted box in a-reveals the motion of vortices (labeled by color) at the interface (blue line). Vortices starting in the bulk can collide and attach to the interface (labeled brown vortex for example); some vortices at the surface detach and move away (green, orange). Others fade away (purple) or split (dark blue splits from light blue vortex in right tile). Data shown here is from a single experiment; results were repeated for a minimum of $N=4$ experiments

active phase. Passive bacteria enter the active phase as they are convected away from the interface; their fraction far from the interface however is small since the overall (initial) size of the passive phase is small compared to the total swarming area. Nevertheless, direct observation of the interface between the active and passive phases and results from PIV illustrating a mixing region between the two phases suggests that the interphase boundary may be represented as a diffuse interface with a finite thickness with the density of active motile bacteria varying very sharply across a boundary layer thickness, We hypothesize that, similarly, the boundary between the passive (immobile) and active (swarming) bacteria may be treated as an diffuse interface possessing an intrinsic time dependent thickness, $w$. We surmise that continuous phase-field order parameters can be used to define a mathematically defined diffuse boundary interface as done in previous investigations involving interface phenomena $^{36}$ and phase transition/separation and damage problems in thermoviscoelastic materials ${ }^{37,38}$.

The active-passive phase field. Here, we utilize two independent scalar order parameters to track and characterize the interface; 
a

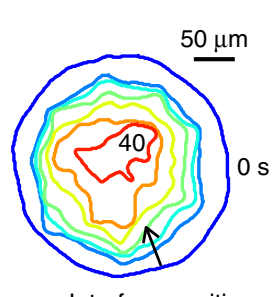

C

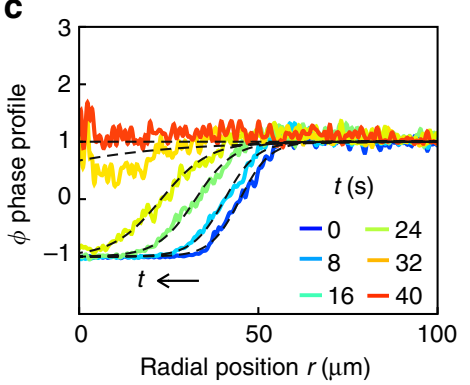

f
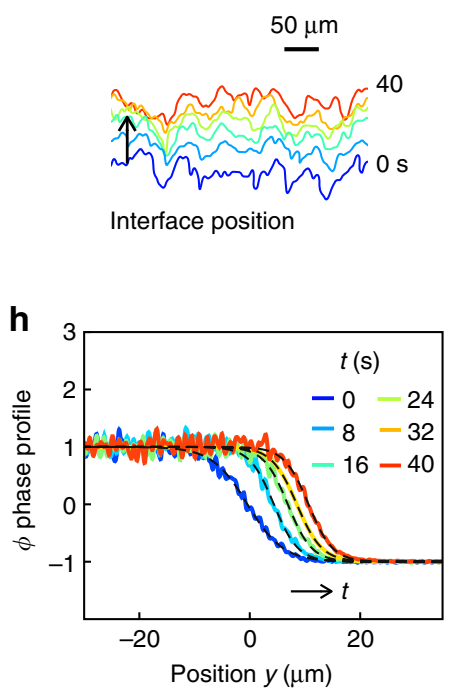

b
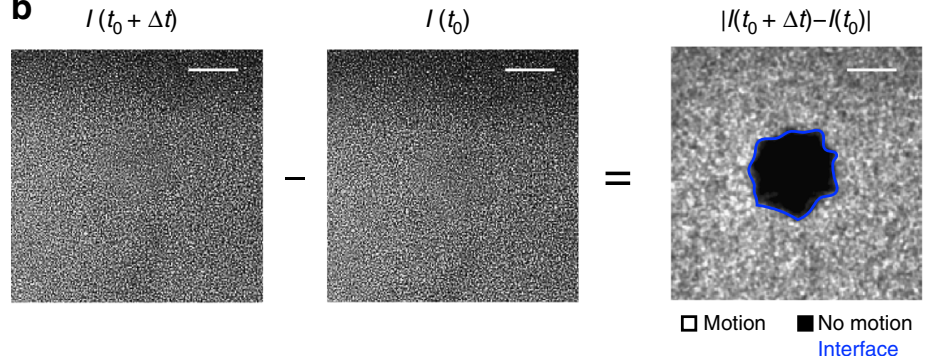

Bacterial speed map

d

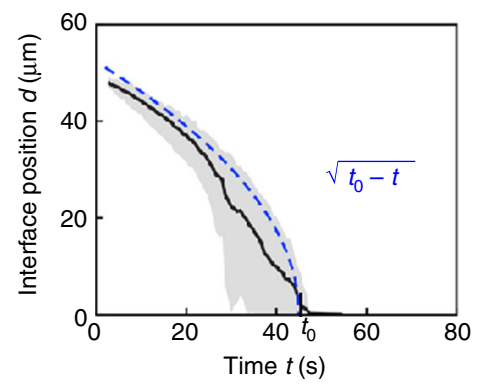

e

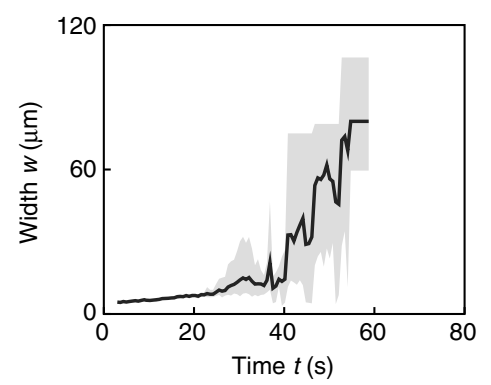

g $\quad I\left(t_{0}+\Delta t\right)$

$I\left(t_{0}\right)$
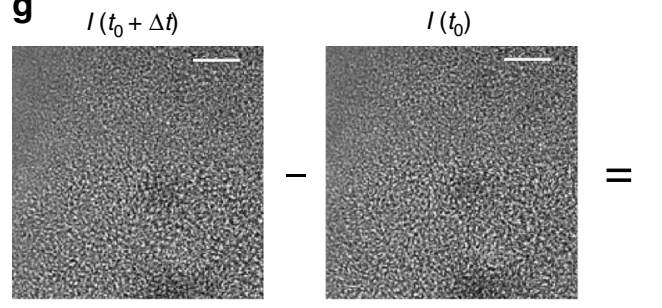

$\left|/\left(t_{0}+\Delta t\right)-I\left(t_{0}\right)\right|$

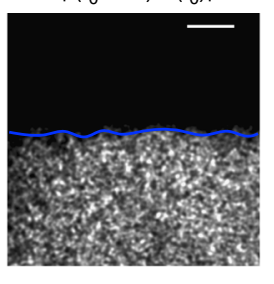

Bacterial speed map

$\square$ Motion

No motion

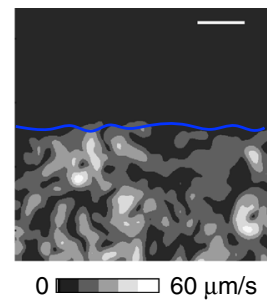

i

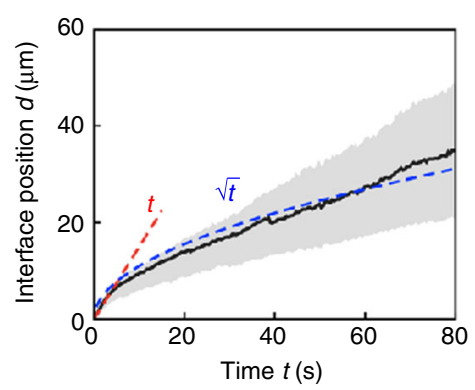

j

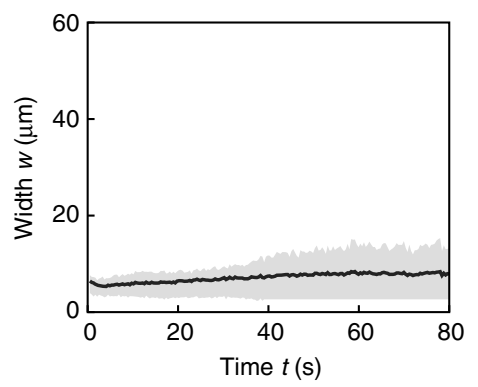

Fig. 2 Growth and structure of the active-passive interface in the bacterial swarm. Active-passive domain boundaries are designed with different initial shapes by varying the geometry of the aperture, such as an octagon [O] (a-e) or half-space $[\mathrm{H}](\mathbf{f}-\mathbf{j})$. a The interface position changes shape as it moves over time. $\mathbf{b}$ The interface position is identified by order parameters based on fluctuations in image intensity, $\phi(\Delta t=0.1 \mathrm{~s})$ and the bacterial speed, $\phi_{v}$ (Methods 4). In a, the boundary correspond to $\phi^{\star}=0$. In $\mathbf{b}$ the boundary is manually traced. c The averaged one-dimensional profiles of the $\phi$-fields smoothly transitions between the active $(\phi=1)$ and passive $(\phi=-1)$ phases. Fits of the data (dashed lines, Eqs. (6) and (7) Methods) yield the mean location $d$ and width $w$ of the interface. $\mathbf{d}, \mathbf{e}$ Parameters $d$ and $w$ are determined for multiple experiments and averaged together as a function of time. Black lines in $\mathbf{d}, \mathbf{e}$ and $\mathbf{i}, \mathbf{j}$ are the averaged results of $N=4$ experiments per condition; gray represents their min to max variation. For the [O] aperture, the mean interface position $d(t)$ (black line, measured radially from the center of the domain) decreases over time and follows the scaling law $d \sim \sqrt{\left(t_{0}-t\right)}$ with $t_{0} \approx 40 \mathrm{~s}$. $\mathbf{f}-\mathbf{j}$ Corresponding results for $[\mathrm{H}]$ aperture. For this case, the interface position $d$ initially follows $d \sim t$ (red line) and then transitions to faster than $d \sim \sqrt{t}$ (blue line) at $t \approx 2 \mathrm{~s}$. Width $w(\mathbf{e}, \mathbf{j})$ ranges from 4 and $10 \mu \mathrm{m}$ and varies little over time-except for the case of the octagon aperture at long times $(t>40 \mathrm{~s})$ as the passive domain dissolves entirely. Black lines in $\mathbf{d}, \mathbf{e}, \mathbf{i}, \mathbf{j}$ are the averaged results of $N=4$ experiments per condition; gray represents their min to max variation. Scale bar, $50 \mu \mathrm{m}$

the first, $\phi$ (Methods 4, Supplementary Note 3) is based on intensity fluctuations between images of the swarm, which are related to bacterial density fluctuations, and the second $\phi_{v}$ is based on the bacterial velocity fields. Each order parameter quantifies the local motion throughout the swarm as shown in Fig. 2: the active phase corresponds to values of +1 and the passive phase, -1 . A transitional interfacial region exists between the two phases; we define the mathematical interface as the locus of points $\mathbf{r}_{s}$ satisfying $\phi\left(\mathbf{r}_{s}, t\right)=0$ (Fig. $2 \mathrm{a}, \mathrm{f}$ ). We find that the results obtained from using the order parameters $\phi$ and $\phi_{v}$ yield essentially the same description of the active-passive interface as seen in the phase-field maps in Fig. 2b, g; here, we present results primarily from $\phi$ based on intensity fluctuations (see Supplementary Fig. 4 for $\phi_{v}$ results).

Our active-passive phase fields possess many of the main features of a propagating, broadening, and coarsening interface. To examine the boundary evolution, we spatially average the phase field into one-dimensional phase profiles, $\phi^{*}$ 
a

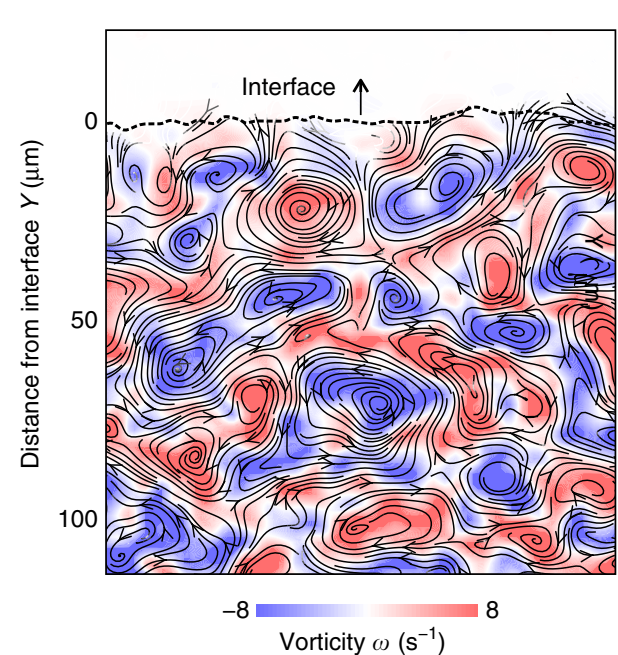

b $\left\langle\omega^{2}\right\rangle\left(\mathrm{s}^{-2}\right)$

$0 \quad 102030$

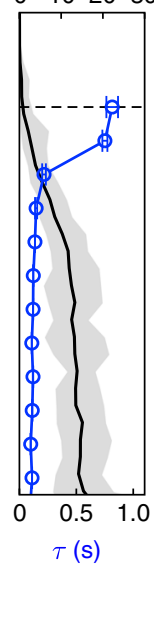

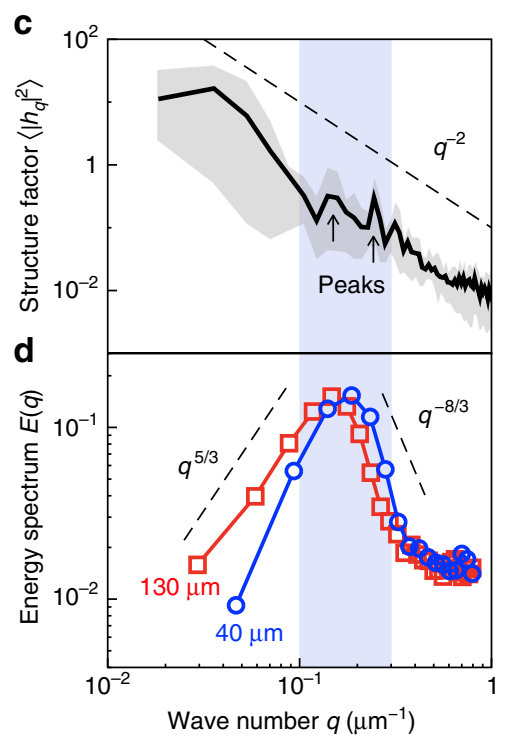

Fig. 3 Interface structure is coupled to collective flow of bacteria. a A snapshot of the bacterial vorticity field, $t=4 \mathrm{~s}$ after exposure with the half-space ( $\mathrm{H}$ ) aperture. $\mathbf{b}$ The strength of the vorticity field, characterized by $\left\langle\omega^{2}\right\rangle$, increases as one moves away from the interface boundary; while the flow decay timescale $\tau$ decreases (blue error bars represent standard error and gray variation represents min to max values gathered from temporal fluctuations in a representative experiment). c The overall decay of the interface static structure factor scales as $\left|\Delta h_{q}\right|^{2} \sim q^{-2}$, yielding an interfacial stiffness $\kappa=0.7 \mu m^{-2}$. The black line represents the averaged results of $N=4$ experiments for the half-plane aperture geometry; gray variation represents min to max. $\mathbf{d}$ The energy spectrum of the flow $E(q)$ (Eq. (9)) is non-monotonic with a peak that shifts to higher values of $q$ close to the interface $(Y \approx 40 \mu \mathrm{m})$ compared with the bulk active phase $(Y \approx 130 \mu \mathrm{m})$; data averaged over $10 \mathrm{~s}$ from a representative sample. The peak in $E(q)$ coincides with peaks in $\left|\Delta h_{q}^{2}\right|$

(Fig. 2c, h, Methods 5). The phase profiles are well captured using fits to hyperbolic tangent functions (Methods 5), which indicates a functional dependence and yields the mean interface position $d$ and interface thickness $w$ over time.

The evolution of the mean interface position $d$ depends on the shape of the aperture (Fig. 2d, i). For the (O) aperture, $d(t)$ provides an area averaged radius of the passive domain that decreases over time, eventually reducing to zero as the whole domain erodes away; in this case, we find $d \sim \sqrt{t_{0}-t}$ (Fig. 2d), with the time at which the passive phase disappears, $t_{0} \approx 40 \mathrm{~s}$. For the $(\mathrm{H})$ aperture (Fig. $2 \mathrm{i})$, the mean interface position propagates faster than $d \sim \sqrt{t}$ for the duration of the experiment.

The interface thickness $w$ ranges from 4 to $10 \mu \mathrm{m}$ ( bacterial length) over most of the dissolution process (Fig. 2e, j). The most significant deviation from this trend is seen for the [O] aperture at relatively long times (Fig. 2e): when the interface width is approximately the radius of the passive phase $(t \approx 40 \mathrm{~s})$, both $w$ and $d$ exhibit large fluctuations and the thickness increases dramatically. Our results show that the interface width is a weak function of time, consistent with a quasi-steady propagating interface. We do observe isolated bacteria-singly or in very small pockets-entering into the passive region at distances more than the interface thickness (Supplementary Movie 4). However, these events are rare, resulting in the averaging procedure yielding a phase-field based interface without overhangs. We interpret $w$ as a correlation length characterizing the gradient in the density of active bacteria.

The active-passive phase fields based on intensity fluctuations $(\phi)$ and bacterial velocity field $\left(\phi_{v}\right)$ yield qualitatively similar results (Supplementary Note 3). For instance, the interface positions are the same (Supplementary Fig. 4). We hypothesize that $w_{v}$ is greater than $w$ (Supplementary Fig. 4 ) because velocity fields vary over vortex $(20 \mu \mathrm{m})$ length scales whereas intensity fluctuations vary over bacterial scales $(5 \mu \mathrm{m})$.

Coupling between the boundary and collective flow of bacteria. Next, we examine how the bacterial flow interacts with the interface. Figure 3a highlights two robust features of the flow near the interface: (i) a gradient in flow perpendicularly across the interface and (ii) an array of size-varying vortical flows. We find that the flow varies in both strength and dynamics. As shown in $3 \mathrm{~b}$, the square of vorticity $\left\langle\omega^{2}\right\rangle$ increases as one moves away from the interface; while the flow decay time $\tau$ decreases (Methods 6), indicating an increase in vortical flow lifetimes, a result similar to recent simulations of active and passive sphere mixtures ${ }^{32}$. Overall, our data suggests a coupling between the interface and flow: the interface plays a stabilizing role on the collective flow while the gradient in bacterial vorticity marks a flux of momentum and energy from the swarming bacteria to the interface, energy that can be used to erode and reform the surface. Consistent with this picture, the interface undergoes displacements through interactions with many fluctuating vortical flows (Methods 6).

To understand the boundary-flow coupling, we calculate the (quasi-static) structure factor of the interface (Fig. 3c) and the two-dimensional energy spectrum of the flow (Fig. 3d). We focus here on experiments using the $[\mathrm{H}]$ aperture, which are not limited by finite time dissolution effects. The static structure factor $\left\langle\left|\Delta h_{q}\right|^{2}\right\rangle$ (Methods 6) decays with wavenumber $q$, punctuated by peaks at $q=0.15$ and $0.22 \mu \mathrm{m}^{-1}$ (Fig. 3c). For $q>0.4 \mu \mathrm{m}^{-1}$, the overall decay scales as $\left\langle\left|\Delta h_{q}\right|^{2}\right\rangle \sim q^{-2}$. To correlate these length scales with the energetic features of the flow, we calculate the twodimensional time-averaged energy spectra $E(q)$ from the bacteria velocity field (Methods 6) for different distances from the interface $Y$. As shown in Fig. 3d, $E(q)$ is non-monotonic with $q$ and depends on $Y$. This non-monotonic behavior is a unique characteristic of active fluids and is attributed to the injection of energy at the level of the bacteria ${ }^{39}$. Compared to dense suspensions of swimming bacteria in microfluidic devices ${ }^{39}$, our measured spectra exhibit similar scalings with $q$ as $q^{5 / 3}$ and $q^{-8 / 3}$ (Fig. 3d) with a peak centered at scales of about 1-2 vortex sizes. The interface also impacts the flow spectra by shifting the peak to higher $q$, indicating a shift to smaller vortices near the interface. 
For passive fluid-fluid interfaces fluctuating due to white noise, equipartition of energy requires that $\left\langle\left|\Delta h_{q}\right|^{2}\right\rangle=\left(\kappa A q^{2}\right)^{-1}$ with $\kappa$ being the stiffness, and $A$ the interface area. Equipartition does not hold in our system. The peak in $E(q)$ (between $0.15-0.2 \mu \mathrm{m}^{-1}$; length scale, $15-20 \mu \mathrm{m})$ appears to overlay peaks in the static structure factor $\left\langle\left|\Delta h_{q}\right|^{2}\right\rangle$ (see Supplementary Fig. 7), suggesting that the interface is shaped at length scales of the flow structures (streamers and vortices). Still, the scaling $\left\langle\left|\Delta h_{q}\right|^{2}\right\rangle \sim q^{-2}$ provides a good description over a range of length scales (Fig. 3c). We use this form to fit the data (Methods 7) and extract an interfacial stiffness $\kappa \approx 0.7 \mu \mathrm{m}^{-2}$. This value is much smaller than the interfacial stiffness of water in air $\left(1.7 \times 10^{7} \mu \mathrm{m}^{-2}\right)$ but similar to phase-separated systems involving colloids ${ }^{40-42}\left(0.1-20 \mu \mathrm{m}^{-2}\right)$. This stiffness is to be interpreted as an effective value of the diffuse interface and inherently accommodates variations in passive bacteria alignment.

Here, the bacterial flow injects energy into the surface at various wave-numbers and frequencies. One way to qualify the energetic features of the flow is by invoking an effective

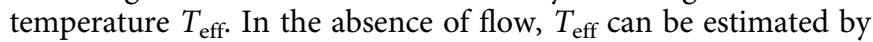
following tracers that sample the system for sufficiently long times ${ }^{43}$. Our swarm features collective flows; $T_{\text {eff }}$ therefore is ambiguous. Nonetheless, using $2 \mu \mathrm{m}$ polystyrene spheres as tracer particles (Methods 7, Supplementary Fig. 10), we estimate $T_{\text {eff }} \approx 2.2 \times 10^{5} \mathrm{~K}$, which yields an apparent surface tension $\kappa k_{\mathrm{B}} T_{\text {eff }} \sim 10 \mathrm{pN} / \mu \mathrm{m}$.

Growth and form of the active-passive interface. In the paradigmatic example-melting of ice-the interface between ice and water propagates with speeds controlled by the temperature field at the interface and heat flux across the interface. For a stationary ice-water boundary, the Gibbs-Thomson relationship provides the relationship between the interface curvature $\mathcal{C}$ and the temperature (equivalently, chemical potential) while the Stefan boundary condition constrains the flux of heat across the interface. For slowly propagating ice-water interfaces, the Gibbs-Thomson relationship requires extension; a linear relationship can be obtained between the local curvature, local interface velocity, and temperature (or chemical potential) 27,28 .

To test for analogous boundary conditions for active-passive interfaces, we visualized how active flows extracted and convected passive particles at the boundary (Fig. $4 \mathrm{a}, \mathrm{b}$ ). We found that some particles move tangentially along the surface before being swept away by the collective flows whereas other particles appear ejected normally from the surface (Supplementary Movie 6), results that together suggests anisotropic caging effects due to bacteria alignment (see Methods 8). Based on our observations, we propose a simple linear relation that captures the main ingredients of the erosion process that connects the interface kinematics to geometry and intensity of activity,

$$
v_{\text {int }}=a_{1}+a_{2} \mathcal{C}+a_{3} \mathcal{X}
$$

with the variable $\mathcal{X}$ standing in for the swarm activity. We chose bacterial speed $|v|, v_{\mathrm{N}}$ (normal component of the bacterial velocity), $v_{\mathrm{T}}$ (tangential component), and vorticity $\omega$ as possible stand-ins for $\mathcal{X}$ and evaluated the fidelity of the fit of Eq. (1) to the experimental data (Methods 8, Supplementary Note 5). We gather these data at multiple points along the interface for varying times throughout the experiment. The data is combined with local measures of the interface velocity and interface curvature and cast into three-dimensional scatter plots (Supplementary Fig. 9). Scatter of the experimental data can be attributed to image processing errors, stochastic noise in bacterial length and speed, and variations in bacterial orientation.
The best collapse of the data to a plane-with statistical significance (Methods 9)-was obtained for the half-space aperture with $v_{\mathrm{N}}$, as shown in Fig. $4 \mathrm{c}$. The values of the coefficients are dependent on the aperture geometry. For the halfplane $[\mathrm{H}]$ aperture, we find $a_{1}=0.4 \mu \mathrm{m} / \mathrm{s}, a_{2}=-2.3 \mu \mathrm{m}^{2} / \mathrm{s}$, and $a_{3}=-2.0$. For the octagonal [O] aperture (Fig. $4 \mathrm{~d}$ ), the presence of a mean curvature shifts the plane, which we find for $v_{\mathrm{N}}$ to be given by $a_{1}=0.02 \mu \mathrm{m} / \mathrm{s}, a_{2}=-1.12 \mu \mathrm{m}^{2} / \mathrm{s}$, and $a_{3}=-0.7$. A possible reason for the slight difference in the coefficients for the two geometries, particularly for $a_{2}$ multiplying the curvature term, is the presence of corners in the octagon geometry that appear to introduce additional streaming flows. Twodimensional projections of the planes are shown in Supplementary Fig. 8. At a qualitative level, the scatter plots in Fig. 4 seems to show a three-way coupling between the interface velocity, bacterial flow, and curvature in a manner reminiscent of the classical Gibbs-Thomson-Stefan boundary conditions in passive systems, but the analogy is only suggestive given the correlation data. We find that the interface velocity is negatively correlated with curvature $\mathcal{C}$; regions of negative curvature move into the passive phase as passive bacteria are extracted from the surface.

\section{Discussion}

We conclude that that dissolution of passive domains within active swarms is a spontaneous self-organized process governed by short-range interactions at the single bacterial level and longrange non-local effects due to collective flow. Three features define the active-passive interface during the erosion process. First, the interphase region acts as a broadening diffuse interface with a well defined position and thickness. Second, the interface is stiff with elastic constants dependent on flow intensity; stronger flows are expected to lower the effective stiffness, allowing for faster erosion. Third, the interface structure is tightly coupled to the statistics of the collective swarming flows. The interphase boundary stabilizes vortices that form parallel to the surface, enabling sustained erosion. Interface erosion processes may be interpreted using an active analog of the classic Gibbs-Thomson-Stefan boundary conditions, although more detailed experiments and analysis need to be performed to firmly establish such connection.

Our results highlight a new finding that the presence of soft deformable boundaries impacts the collective motion of swarming flows by stabilizing vortices and increasing flow correlation times. One possible reason may be related to the mechanism of how the active particles interact with and dislodge passive particles. It may be easier for the active bacteria to realign and stay moving tangentially to the surface versus normally, until they leave the interface due to either rotational diffusion (suppressed here due to the high density) or collective reorientation. As long as the radius of curvature (macroscopic) of the interface is larger than the bacteria size, the bacterium will spend more time aligned tangential to the interface and thus keep moving. The strong effect of the interface on the bacterial flow may be surprising, given that bacterial swarming motility is the most rapid form of surface motility and emerges naturally in crowded environments. Our results here indicate that the presence of a boundary layer and associated constraints on the fluid velocity may be the important mechanical feature through which the interface influences bacterial motility.

Interfaces separating cells of differing motility are a common motif in natural habitats, including biofilms, where swimmers, spores, and other non-motile phenotypes segregate into different domains ${ }^{44}$. Starting with light-induced boundaries in a model system of Serratia marcescens swarms, we identified important physical mechanisms that underlie the erosion of such active interfaces and the two way coupling between interface propagation and emergent flows in the active region. We anticipate that 
a
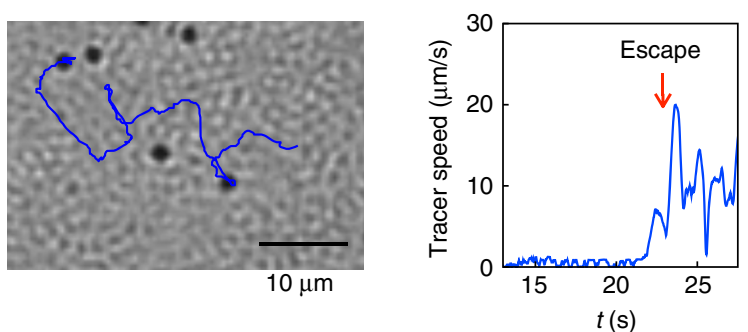

b

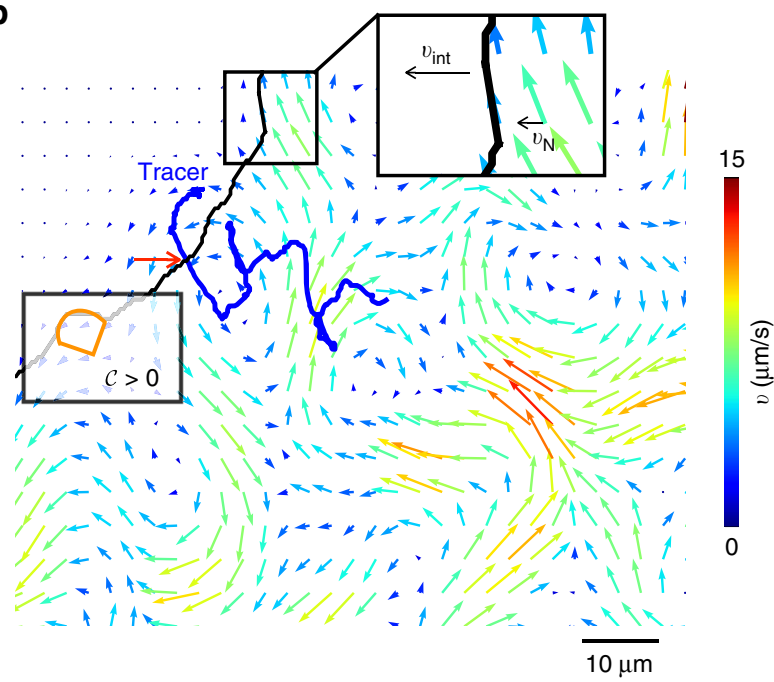

C

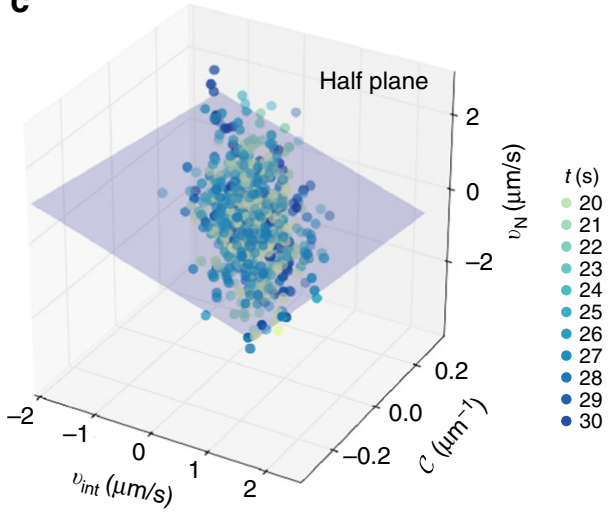

d

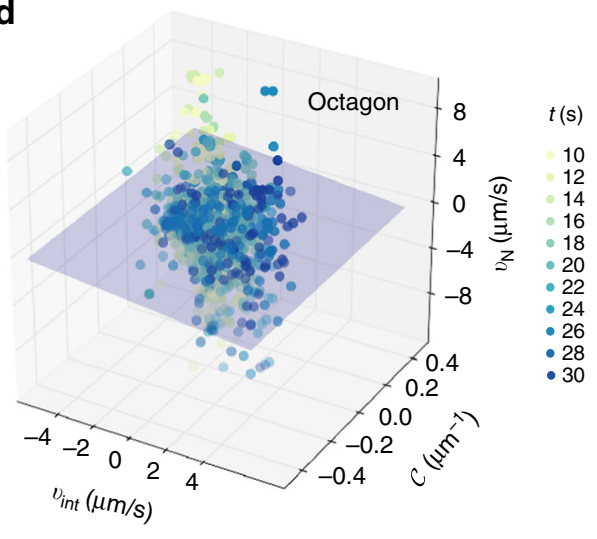

Fig. 4 Evolution of the active-passive interface follows an active analog to classical Gibbs-Thomson-Stefan conditions. a Particle motion at the interface. A representative trajectory of a $2 \mu \mathrm{m}$ polystyrene tracer particle reveals how the swarm extracts particles from the passive domain. First, particles in the passive phase caged-in by their neighbors do not move. Second, when the diffuse interphase is roughly a velocity correlation length $O\left(w_{v}\right)$ away, the trapped particle starts to fluctuate, as seen in its speed over time. Third, agitations induced by the active particles dislodge the jammed tracer from the cage of neighboring passive particles. For the tracer in $\mathbf{b}$ the flow field moves the particle tangentially along the interface (Supplementary Movie 6). Finally, the particle escapes the passive domain as normal streaming flows between adjacent vortices pull the particle away, primarily perpendicularly from the interface. This escape correlates with a rapid increase in particle speed; once inside the swarm, the speed of the tracer fluctuates (red arrow). The schematic defines the sign of the curvature and normal bacterial velocity. $\mathbf{c}, \mathbf{d}$ Local interface velocity $v_{\text {int }}$ correlates with the interface curvature $\mathcal{C}$ and the normal component of the collective bacterial velocity $v_{\mathrm{N}}$ at the interface. Data for $v_{\text {int }}, \mathcal{C}$, and $v_{\mathrm{N}}$ collapses toward a plane for $\mathbf{c}$ half-plane $[\mathrm{H}]$ and $\mathbf{d}$ octagonal [O] aperture geometries; data shown here from representative experiments and gathered at $3 \mu \mathrm{m}$ spacings across the interface over a $10-20 \mathrm{~s}$ interval (Supplementary Note 5). The collapse of the data unto plane suggests the existence of an active analog of the classical Gibbs-Thomson-Stefan boundary conditions. Spatiotemporal analysis shown here was verified for a minimum of $N=2$ experiments per condition

these physical mechanisms underlie segregation and pattern formation in active biological and synthetic settings with emergent flows. Naturally, the next step would be to explore how extracellular polymers-which are implicated in both single cell swimming 45,46 and collective biofilm expansion ${ }^{47}$-impact boundary dynamics in microbial environments. Our results also motivate additional experimental and theoretical studies to explore if boundary conditions governing interface growth and form of passive interfaces may be extended or adapted to active interfaces.

\section{Methods}

Preparation of bacterial swarms and imaging. We use swarming Serratia marcescens cultured on on agar substrates. The agar is prepared by dissolving $1 \mathrm{w} \%$ Bacto Tryptone, $0.5 \mathrm{w} \%$ yeast extract, $0.5 \mathrm{w} \% \mathrm{NaCl}$, and $0.6 \mathrm{w} \%$ Bacto Agar in $\mathrm{ddH}_{2} \mathrm{O}$. This is melted and poured into petri dishes after addition of $2 \mathrm{w} \%$ of glucose solution (25 w\%). Once the agar cools and solidifies, Serratia marcescens (strain ATCC 274, Manassas, VA) from frozen glycerol stocks are inoculated on the agar plates and incubated at $34^{\circ} \mathrm{C}$. Bacterial colonies form at the inoculation sites and grow outward on the agar substrate, away from the inoculation site. Experiments are performed $12-16 \mathrm{~h}$ after inoculation; the bacteria are imaged with the free surface facing down with an inverted microscope. The bacteria were imaged with an inverted Nikon microscope Eclipse Ti-U with a Nikon 20x
$(\mathrm{NA}=0.45)$ and Nikon $60 \mathrm{x}(\mathrm{NA}=0.7)$ objective. Images were gathered at either 60 or 125 frames per second with a Photron Fastcam SA1.1 camera.

Bacterial velocity fields from PIV. Bacterial velocity fields, $\mathbf{v}(\mathbf{r}, t)$, were extracted at $3 \mu \mathrm{m}$ intervals from videos using particle image velocimetry (PIV, PIVLab, Supplementary Note 2). The velocity fields reveal complex transient collective flows at the expanding edge of the colony. Selecting regions of the swarm spanning 100 to $500 \mu \mathrm{m}$ from the colony edge-where the swarming behavior appears strongest -we calculated the spatial and temporal velocity correlation functions, $C_{r}(\Delta r)$ and $C_{t}(\Delta t)$ using

$$
C_{r}(\Delta r)=\left\langle\frac{\mathbf{v}\left(r_{0}\right) \cdot \mathbf{v}\left(r_{0}+\Delta r\right)}{\left|\mathbf{v}\left(r_{0}\right)\right|^{2}}\right\rangle,
$$

and

$$
C_{t}(\Delta t)=\left\langle\frac{\mathbf{v}\left(t_{0}\right) \cdot \mathbf{v}\left(t_{0}+\Delta t\right)}{\left|\mathbf{v}\left(t_{0}\right)\right|^{2}}\right\rangle .
$$

Here, the brackets denote and average over reference times $t_{0}$ and reference positions $r_{0}$. Pre-exposure, the swarming behavior does not appear to be biased in any particular direction (the swarming speeds are much larger than the speed of the advancing colony front); hence, scalar function defined by (1) and (2) suffice to characterize the flow. From these measurements, we extract a characteristic vortex 
size of $20 \mu \mathrm{m}$ and vortex (residence or lifetime) timescale of $0.24 \mathrm{~s}$ (Supplementary Note 2, Supplementary Fig. 1).

Exposure to high intensity UV light. To create a passive phase of immobile bacteria, we used standard fluorescence microscope optics to expose cells to high intensity light. The light source is an unfiltered mercury lamp, which has a widespectrum that includes a significant amount of UV light $(100-400 \mathrm{~nm})$. The response of bacteria to light depends on the wavelength, intensity, and duration of the light ${ }^{48}$ and is affected by photosensitizers ${ }^{48,49}$. High intensity light $\geq 200 \mathrm{~mW} /$ $\mathrm{cm}^{2}$ at wavelengths $390-530 \mathrm{~nm}$ can lead to increased tumbling, slow swimming, and eventual irreversible paralysis ${ }^{48,49}$. It is hypothesized that paralysis may be due to flagellar motor damage caused by photosensitizing flavins and dyes that are present in growth media such as LB Broth. Experiments ${ }^{50}$ with Bacillus subtilis also suggest that photosensitizers can be used to reversibly or irreversibly affect collective motility. Cell death occurs from photodynamic action and UV light-induced DNA damage.

Consistent with previous experiments ${ }^{48-50}$, we found that for swarming Serratia marcescens passivation was not immediate and sometimes reversible; yet for sufficiently large light intensities and exposure times they were rendered permanently passivated. We selected an exposure time of $60 \mathrm{~s}$ and light intensity of $I=370 \mu \mathrm{W}$ (measured at $535 \mathrm{~nm}$ ) to reproducibly immobilize Serratia marcescens (Supplementary Note 1).

Phase-field order parameters identify interface. We use two scalar order parameters, $\phi$ and $\phi_{v}$, based on a phase-field description of the interface region between the active (motile) and passive (non-motile) domains to define, identify, locate, and characterize the interface boundary (see also Supplementary Note 3).

(I) From intensity fluctuations: The first scalar order parameter, $\phi$ is computed from intensity fluctuations, $\Delta I$, defined by the difference in image intensities taken at $0.1 \mathrm{~s}$ intervals of the bacterial swarm (Supplementary Note 3). Treating the intensity fluctuation as a measure of the fluctuations in the bacteria density, we define the order parameter at location $r$ by

$$
\phi(\mathbf{r}, t)=\frac{2|\Delta I(\mathbf{r}, t)|-\left|\Delta I_{\mathrm{A}}(t)\right|-\left|\Delta I_{\mathrm{P}}(t)\right|}{\left|\Delta I_{\mathrm{A}}(t)\right|-\left|\Delta I_{\mathrm{P}}(t)\right|} .
$$

where $\left|\Delta I_{\mathrm{A}}(t)\right|$ and $\left|\Delta I_{\mathrm{P}}(t)\right|$ are the intensity fluctuations of the active and passive phases far from the interface. Here, $-1 \leq \phi \leq+1$, with -1 corresponding to the completely passive phase and 1 corresponding to the completely active phase.

The interfacial region is fuzzy with finite thickness due to the intermingling of active and passive bacteria. Following classical phase-field approaches, we mathematically define the interface location as the locus of points satisfying $\phi=0$.

(II) From velocity fields through PIV: The second order parameter $\phi_{v}$ is based on the velocity fields calculated from PIV (Supplementary Note 3, Supplementary Fig. 3). We define

$$
\begin{aligned}
& \text { Fig. 3). We define } \\
& \phi_{v}(\mathbf{r}, t)=\frac{2 v^{2}(\mathbf{r}, t)-v_{\mathrm{A}}^{2}-v_{\mathrm{P}}^{2}}{v_{\mathrm{A}}^{2}-v_{\mathrm{P}}^{2}}
\end{aligned}
$$

where $v_{\mathrm{A}}^{2}$ and $v_{\mathrm{P}}^{2}$ are velocities of the active and passive phases far from the interface and $r$ is the position of velocity vectors sampled at $3 \mu \mathrm{m}$ intervals. Again we have the bounds $-1 \pm \phi_{v} \leq+1$ with the interface location defined as the locus of points satisfying $\phi_{v}=0$.

Position and thickness of the diffuse interface. The boundary separating the active and passive phases resembles a propagating and broadening interface. To analyze this behavior, we fit the order parameter profiles to the classical form for a quasi-equilibrium diffuse, moving interface. We use the order parameter fields extracted from intensity fluctuations (Fig. 2) and PIV data (Supplementary Note 3) to obtain averaged one-dimensional phase profiles.

For experiments with the $[\mathrm{H}]$ aperture, we choose cartesian coordinates $(x, y)$ with the $y=0$ line aligned with the edge of the exposure. For times post-exposure, fields are averaged in the $x$ direction to obtain $\phi^{*}(y, t)$. Data from experiments then is fit to

$$
\begin{aligned}
\phi^{*} & =\tanh \left[\frac{y-d(t)}{w(t)}\right], \text { and } \\
\phi_{v}^{*} & =\tanh \left[\frac{y-d_{v}(t)}{w_{v}(t)}\right] .
\end{aligned}
$$

Here $d(t)$ and $d_{v}(t)$ denote the interface location and $w(t)$ and $w_{v}(t)$ denote the diffuse interface thickness.

In experiments using the $[\mathrm{O}]$ exposure, we define a polar co-ordinate system with center at the center of the passive phase and obtain an azimuthally averaged, radially dependent value of $\phi^{*}(r, t)$. Reduced data is then fit to the forms

$$
\begin{gathered}
\phi^{*}=\tanh \left[\frac{r-d(t)]}{w(t)}\right], \text { and } \\
\phi_{v}^{*}=\tanh \left[\frac{r-d_{v}(t)}{w_{v}(t)}\right]
\end{gathered}
$$

where now $d(t)$ is the averaged radius of the passive domain. Since $r=0$ is the center of the passive region, Eq. (7) will exhibit significant errors for times close to dissolution $(d \leq w)$. The length scale over which $\phi$ varies sharply, $w(t)$ yields the density correlation length characterizing the penetration of active bacteria into the passive phase. The length scale over which $\phi_{v}$ varies sharply, $w_{v}(t)$ yields the momentum penetration length - the length over which passive bacteria are pushed around by the active bacteria without being completely dislodged.

Interface-flow coupling. To compare the structural features of the collective flow with the features of the interface, we calculated the static structure factor of the interface after suitably averaging fluctuations on the order of bacterial lengths. Focusing on experiments with the $[\mathrm{H}]$ aperture, we compute the height fluctuation $\Delta h(t, x)=h(t, x)-d(t)$, where $h(t, x)$ is the interface position interpolated as a function of the arc-length co-ordinate $x$ and $d(t)$ is the mean interface position. Next, the wavenumber dependent Fourier modes of the height fluctuations, $\Delta h_{q}(t)$, was obtained as

$$
\Delta h_{q}(t)=\frac{1}{\mathcal{L}_{x}} \int_{0}^{\mathcal{L}_{x}} \Delta h(x, t) e^{-i q x} d x .
$$

Here, the wavenumber is $q=n \pi / \mathcal{L}_{x}$ where $\left.\mathcal{L}_{x} \approx 200 \mu \mathrm{m}\right)$. We varies $q$ from $0.015 \mu \mathrm{m}^{-1}$, which corresponds to the system size, to $1.0 \mu \mathrm{m}^{-1}$, which corresponds to approximately $3 \mu \mathrm{m}$ ( half a bacterial length). The static structure factor was determined as the temporal-average of the Fourier mode magnitude square, $\left\langle\left|\Delta h_{q}\right|^{2}\right\rangle$.

The energy transfer to the surface comes from the flux of momentum flowing into the passive domain from the active domain as active bacteria invade and erode the interface.

The two-dimensional energy spectra of the flow is defined here through the PIV-velocity fields as

$$
E(q)=\frac{q}{2 \pi} \int d \mathbf{R} e^{-i \mathbf{q} \cdot \mathbf{R}}\left\langle\mathbf{v}\left(t, r_{0}\right) \cdot \mathbf{v}\left(t, \mathbf{r}_{0}+\mathbf{R}\right)\right\rangle,
$$

where the brackets here denote averages over $\mathbf{r}_{0}$. For the near-interface case, we calculate $E(q)$ within an $[80 \times 80] \mu \mathrm{m}^{2}$ area, adjacent to the interface ( $Y$ ranging from $0-80 \mu \mathrm{m}$; average distance $40 \mu \mathrm{m}$ ). For the far from interface case, we calculate $E(q)$ within an $[100 \times 100] \mu \mathrm{m}^{2}$ area with $Y$ ranging from 80 to $180 \mu \mathrm{m}$ We varied $q$ from $\sim 0.03$ to $1.0 \mu \mathrm{m}^{-1}$, corresponding to approximately the size of the region $(80 \mu \mathrm{m})$ and to the bacterial velocity field spacing $(3 \mu \mathrm{m})$, respectively. For the $[\mathrm{H}]$-aperture results (Fig. 3 ), the interface position is a flat propagating line, and $Y$ is the distance normal to this line.

We calculated the autocorrelation function of the boundary position (using the $[\mathrm{H}]$-aperture), defined here as

$$
C_{\text {int }}(\Delta t)=\frac{\left\langle\Delta h\left(t_{0}\right) \Delta h\left(t_{0}+\Delta t\right)\right\rangle}{\left\langle\left|\Delta h\left(t_{0}\right)\right|^{2}\right\rangle},
$$

where the correlation is averaged over reference locations and reference times $t_{0}$. The data is fit to a single decaying exponential, yielding a characteristic decay time $\tau$ of $16 \mathrm{~s}$ (Supplementary Fig. 9). For characterize the bacterial flow, we measure the normalized spatial correlation of the velocity director $\mathbf{v}$ (from PIV) as a function of time $\Delta t$ and the distance normal to the interface $Y$, as given by

$$
C_{\text {flow }}(\Delta t, Y)=\frac{\left\langle\mathbf{v}\left(t_{0}, Y\right) \mathbf{v}\left(t_{0}+\Delta t, Y\right)\right\rangle}{\left\langle\mathbf{v}\left(t_{0}, Y\right)^{2}\right\rangle}
$$

where the correlation is averaged over reference locations and reference times $t_{0}$. The flow correlations are also fit to a single decaying exponential to determine characteristic flow time scales as a function of $Y$ (Supplementary Fig. 9).

Interfacial stiffness and effective temperature. To extract an effective interfacial stiffness $\kappa$, we fit the structure factor in Fig. $3 c$ to $\left(\kappa A q^{2}\right)^{-1}$, assuming that the interfacial area is $h \times L_{X}$, where $h=1 \mu \mathrm{m}$ is the cell width (since the swarm is approximately a monolayer thick ${ }^{5}$ ) and $L_{X}$ is the length of the observed interface, $200 \mu \mathrm{m}$.

To estimate an effective temperature of the swarm, we use two micron polystyrene spheres as probes of the swarming flows in the active region of the colony (Supplementary Fig. 10). The polystyrene particles are cleaned by centrifugation and then suspended in a buffer solution $(67 \mathrm{mM} \mathrm{NaCl}$ aqueous solution) with a small amount of surfactant (Tween $20,0.03 \%$ by volume). A small aliquot of this particle solution $(20 \mu \mathrm{L})$ is gently pipetted unto the bacterial colony in a location where expanding colony front meets the agar. After the polystyrene particles are introduced, we allow the colony to settle for 5 min before imaging. We do not observe any change in the behavior of the swarming bacteria due to the 
addition of these particles at $0.8 \%$ area fraction. We track the particle positions over time using standard particle tracking techniques. We define the (time-averaged) particle speed as the particles displacement (in two dimensions) over a $1 \mathrm{~s} \mathrm{time}$ interval, allowing tracers to sample multiple vortex structures (characteristic lifetimes $\sim 0.1 \mathrm{~s}$ ). The particle speed distributions seem to follow a $2 \mathrm{D}$ Maxwell-Boltmann distribution (Supplementary Fig. 10b), $p(v)=v_{m}\left(k_{\mathrm{B}} T_{\text {eff }}\right)^{-1} \exp \left(-m v^{2} / 2 k_{\mathrm{B}} T_{\text {eff }}\right)$, where $m$ is the mass of the polystyrene particle, $k_{\mathrm{B}}$ is the Boltzmann constant, and $T_{\text {eff }} \approx 2.2 \times 10^{5} \mathrm{~K}, \sim 700$ times the thermal temperature $(293 \mathrm{~K})$. This effective temperature is to be interpreted as a mixture temperature purely due to the energy in the swarming collective flows.

Effects of curvature and flow on interface speed. Does an approximate linear relationship between the interface speed, curvature, and a characteristic of the bacterial flow exist for small propagation speeds? Such a relationship may be viewed then as an active analog of the classical Gibbs-Thomson-Stefan boundary conditions for passive systems. Swarming provides the impetus for the motion and keeps the active-passive interface always out of equilibrium. We can directly gauge the intensity of the flow and its activity via measurements such as PIV or particle tracking methods. This is preferable to using temperature or chemical potential that cannot be measured directly and exact forms of which are a matter of debate ${ }^{10,29,30}$.

To test for correlations between the interface speed, curvature and flow speeds we assume that two independent processes act on the interface leading to erosion and remodeling. The first arises from self-propulsion based interactions at the single bacteria level and is proportional to density of active bacteria $\rho_{\mathrm{A}}$. The second acts at longer spatiotemporal scales and accounts for hydrodynamic interactions and collective flow.

With this picture in mind, we look for a minimal linear description of the interface speed (a vector) by constructing linear terms from the relevant vectors and tensors that impact these mechanisms. Let $v_{\text {int }}(\mathbf{r})$ be the interface velocity at location $\mathbf{r}$ on the interface. Relevant vectors that are expected to influence the interface speed are (A) geometric features of the interface, that is the normal at this location $\mathbf{n}(\mathbf{r}, t)$ and the tangent vector $\mathbf{t}(\mathbf{r}, t)$, and (B) features of the collective flow -namely the (collective) bacterial velocity field $\mathbf{v}$. Relevant tensors that are expected to influence the interface speed are (A) gradients of $\mathbf{v}$ and the alignment tensor $\mathbf{Q}$ that quantifies the orientation of the jammed passive bacteria in the neighborhood of $\mathbf{r}$.

To simplify matters further, and motivated by the mechanisms by which passive bacteria are eroded from the interface, we assume that tangential and normal components of the collective flow are more important than higher order gradients in $\mathbf{v}$. We emphasize however that vorticity and shear gradients in $\mathbf{v}$ influence these normal and tangential velocity components. In two dimensions and at a scaling level the vorticity $\omega \sim|v| / w_{v}$. Ignoring quadratic terms and higher order gradient terms in $\nabla \mathbf{n}, \nabla \mathbf{t}, \nabla \mathbf{v}$, and $\nabla \mathbf{Q}$ we then write

$$
\begin{gathered}
v_{\text {int }} \mathbf{n} \approx \rho_{\mathrm{A}} \mathbf{A}_{1}+\rho_{\mathrm{A}} \mathbf{A}_{2}(\nabla \cdot \mathbf{n})+\rho_{\mathrm{A}} \beta_{1}(\mathbf{n} \cdot \mathbf{Q} \cdot \mathbf{n}) \mathbf{v}+ \\
\rho_{\mathrm{A}} \beta_{2}(\mathbf{n} \cdot \mathbf{Q} \cdot \mathbf{t}) \mathbf{v}+\rho_{\mathrm{A}} \beta_{3}(\mathbf{t} \cdot \mathbf{Q} \cdot \mathbf{t}) \mathbf{v}+\rho_{\mathrm{A}} \beta_{4}(\mathbf{t} \cdot \mathbf{Q} \cdot \mathbf{n}) \mathbf{v}+ \\
\rho_{\mathrm{A}}\left(\beta_{5} \mathbf{v} \cdot \mathbf{Q} \cdot \mathbf{n}+\beta_{6} \mathbf{n} \cdot \mathbf{Q} \cdot \mathbf{v}+\beta_{7} \mathbf{t} \cdot \mathbf{Q} \cdot \mathbf{v}+\beta_{8} \mathbf{v} \cdot \mathbf{Q} \cdot \mathbf{t}\right)
\end{gathered}
$$

with $\mathbf{A}_{1}$ and $\mathbf{A}_{2}$ being functionals of $\mathbf{Q}, \mathbf{n}$, and $\mathbf{t}$ with $\mathbf{Q}$ here comprising density variations and alignment effects in tandem and $\beta_{k}$ with $k=1-8$ being $O(1)$ constants permitted by linearity. The first term on the right hand side reflects local density driven self-propulsion effects present even in the absence of curvature or collective motion. The second curvature term reflects variations in the erosion rate due to geometric effects and bacteria preference to reside near the surface. The other terms describe erosion driven by the collective flow and include normal and tangential velocity contributions. Equation (12) as written cannot be used directly to interpret our data as we do not measure or visualize the alignment field of bacteria in the passive region.

To gain insight into the form of this linear relationship, we studied the trajectories of $2 \mu \mathrm{m}$ polystyrene tracer particles trapped in the passive phase to identify the sequence of mechanistic events leading to their eventual extraction (Fig. 4a). From observations on dozens of tracers, we find that erosion occurs due to initially trapped passive particles being dislodged from their neighbors, then moving parallel to the surface as they are sheared by active bacteria (Supplementary Movie 6), and eventually escaping and leaving that passive domain due to normal streaming flows that occur between vortices at the interface (Fig. 4a). Guided by these observations of tracer particles escaping from the passive phase (Fig. 4a), we further deduce that anisotropic caging effects result in tangential and normal swarm velocities to affect the dislodgment of the passive particles differently. At the simplest level this incorporates the non-isotropic nature of $\mathbf{Q}$. Incorporating these ideas, we write

$$
v_{\text {int }} \approx \rho_{\mathrm{A}}\left(\alpha_{1}+\alpha_{2} \mathcal{C}+\alpha_{3}(\mathbf{v} \cdot \mathbf{n})+\alpha_{4}(\mathbf{v} \cdot \mathbf{t})\right) .
$$

Deviations from this form can be attributed to non-linear effects involving curvature and flow, neglect of alignment effects in the passive phase, and density variations in the active phase. While we expect $\alpha_{1}$ (and thus $a_{1}=\rho_{\mathrm{A}} \alpha_{1}$ ) to be zero in the absence of flow and curvature, we retain this as a fitting constant as higher order terms and the colony front velocity may lead to a net interface propagation.
Statistical analysis. Each experiment was performed a minimum of $N=4$ times for each experimental condition. To test whether the data in Fig. 4c, d could be fit to the equation of a plane (Equation (1) with $\chi$ being substituted as either $v, v_{\mathrm{N}}, v_{\mathrm{T}}$ or $\omega$ ), we performed ordinary least square regression analysis to yield the fitting parameters $a_{1}, a_{2}$, and $a_{3}$ (Eq. (1)); a $t$-test with two tails at the $95 \%$ confidence interval was used to confirm statistical differences between the null hypothesis and the value of each parameter. Results were considered statistically significant when $p$-value was $<0.05$.

\section{Data availability}

All relevant data and in-house programs used in the data analysis are available from the authors.

Received: 5 October 2018 Accepted: 20 November 2018 Published online: 18 December 2018

\section{References}

1. Harshey, R. M. Bacterial motility on a surface: many ways to a common goal. Annu. Rev. Microbiol. 57, 249-273 (2003).

2. Kearns, D. B. A field guide to bacterial swarming motility. Nat. Rev. Microbiol. 8, 634-644 (2010).

3. Alberti, L. \& Harshey, R. M. Differentiation of Serratia marcescens 274 into swimmer and swarmer cells. J. Bacteriol. 172, 4322-4328 (1990).

4. Steager, E. B., Kim, C. B. \& Kim, M. J. Dynamics of pattern formation in bacterial swarms. Phys. Fluids 20, 073601 (2011).

5. Darnton, N. C., Turner, L., Rojevsky, S. \& Berg, H. C. Dynamics of bacterial swarms. Biophys. J. 98, 2082-2090 (2010).

6. Verstraeten, N. et al. Living on a surface: swarming and biofilm formation. Trends Microbiol. 16, 496-506 (2008).

7. Kim, W., Killam, T., Sood, V. \& Surette, M. G. Swarm-cell differentiation in Salmonella enterica serovar Typhimurium results in elevated resistance to multiple antibiotics. J. Bacteriol. 185, 3111-3117 (2003).

8. Butler, M. T., Wang, Q. \& Harshey, R. M. Cell density and mobility protect swarming bacteria against antibiotics. Proc. Natl Acad. Sci. USA 107, 3776-3781 (2010).

9. Marchetti, M. et al. Hydrodynamics of soft active matter. Rev. Mod. Phys. $\mathbf{8 5}$, 1143 (2013).

10. Patteson, A. E., Gopinath, A. \& Arratia, P. E. Active colloids in complex fluids. Curr. Opin. Colloid Interface Sci. 21, 86-96 (2016).

11. Hall-Stoodley, L., Costerton, J. W. \& Stoodley, P. Bacterial biofilms: from the natural environment to infections diseases. Nat. Rev. Microbiol. 2, 95-108 (2004)

12. Scarpa, E. \& Mayor, R. Collective cell migration in development. J. Cell. Biol. 212, 143-155 (2016).

13. Vitorino, P. \& Meyer, T. Modular control of endothelial sheet migration. Genes Dev. 22, 3268 (2008).

14. Ben-Jacob, E., Cohen, I. \& Gutnick, D. L. Cooperative organization of bacterial colonies: from genotype to morphotype. Annu. Rev. Microbiol. 52, 779-806 (1998).

15. Krishna Vedula, S., Ravasio, A., Lim, C. T. \& Ladoux, B. Collective cell migration: a mechanistic perspective. Physiology 28, 370-379 (2013).

16. Ingham, C. J., Kalisman, O., Finkelshtein, A. \& Ben-Jacob, E. Mutually facilitated dispersal between the nonmotile fungus Aspergillus fumigatus and the swarming bacterium Paenibacillus vortex. Proc. Natl Acad. Sci. USA 108, 19731-19736 (2011)

17. Finkelshtein, A., Roth, D., Jacob, E. B. \& Ingham, C. J. Bacterial swarms recruit cargo bacteria to pave the way in toxic environments. mBio 6, e00074-00015 (2015).

18. Gibbs, K. A., Urbanowski, M. L. \& Greenberg, E. P. Genetic determinants of self identity and social recognition in bacteria. Science 321, 256-259 (2008).

19. Budding, A. E. et al. The Dienes phenomenon: competition and territoriality in swarming proteus mirabilis. Proc. Natl Acad. Sci. USA 107, 13626-13630 (2010).

20. Woodward, D. E. et al. Spatio-temporal patterns generated by Salmonella typhimurium. Biophys. J. 68, $4052-4057$ (1995).

21. Cates, M. E., Marenduzzo, D., Paonabarraga, I. \& Tailleur, J. Arrested phase separation in reproducing bacteria creates a generic route to pattern formation. Proc. Natl Acad. Sci. USA 107, 11715-11720 (2010).

22. Ghosh, P., Mondal, J., Ben-Jacob, E. \& Levine, H. Mechanically-driven phase separation in a growing bacterial colony. Proc. Natl Acad. Sci. USA 112, E2166-E2173 (2014).

23. Schwarz-Linek, J. et al. Phase separation and rotor self-assembly in active particle suspensions. Proc. Natl Acad. Sci. USA 109, 4052-4057 (2012).

24. Palacci, J. et al. Living crystals of light-activated colloidal surfers. Science 339, 936-940 (2013) 
25. Takatori, S., De Dier, R., Vermant, J. \& Brady, J. Acoustic trapping of active matter. Nat. Commun. 7, 10694 (2015).

26. Bray, A. J. Coarsening dynamics of phase-separating systems. Philos. Trans. A Math. Phys. Eng. Sci. 15, 781-791 (2003).

27. Langer, J. S. Instabilities and pattern formation in crystal growth. Rev. Mod. Phys. 52, 1 (1980).

28. Back, J. M., McCue, S. W. \& Moroney, T. J. Including nonequilibrium interface kinetics in a continuum model for melting nanoscaled particles. Sci. Rep. 4, 7066 (2014).

29. Takatori, S. C., Yan, W. \& Brady, J. F. Swim pressure: stress generation in active matter. Phys. Rev. Lett. 113, 028103 (2014).

30. Solon, A. P. et al. Pressure is not a state function for generic active fluids. Nat. Phys. 11, 673-678 (2015).

31. Lushi, E., Wioland, H. \& Goldstein, R. E. Fluid flows created by swimming bacteria drive self-organization in confined suspensions. Proc. Natl Acad. Sci. USA 111, 9733-9738 (2014).

32. Wysocki, A., Winkler, R. G. \& Gompper, G. Propagating interfaces in mixtures of active and passive Brownian particles. New J. Phys. 18, 12 (2016).

33. Gopinath, A., Armstrong, R. C. \& Brown, R. A. Observations on the eigenspectrum of the linearized Doi equation with application to numerical simulations of liquid crystal suspensions. J. Chem. Phys. 121, 6093 (2004).

34. Sokolov, A., Rubio, L. D., Brady, J. F. \& Aranson, I. S. Instability of expanding bacterial droplets. Nat. Commun. 7, 1322 (2018).

35. Sokolov, A. \& Aranson, I. S. Rapid expulsion of microswimmers by a vortical flow. Nat. Commun. 9, 11114 (2016).

36. Gopinath, A., Armstrong, R. C. \& Brown, R. A. Second order sharp-interface and thin-interface asymptotic analyses and error minimization for phase-field descriptions of two-sided dilute binary alloy solidification. J. Cryst. Growth 291, 272 (2006).

37. Rocca, E. \& Rossi, R. Entropic solutions to a thermodynamically consistent PDE system for phase transitions and damage. SIAM J. Math. Anal. 47, 2519-2586 (2015).

38. Heinemann, C., Rocca, E. \& Rossi, R. A temperature-dependent phase-field model for phase separation and damage. Arch. Rat. Mech. Anal. 225, 177-247 (2017).

39. Wensink, H. H. et al. Meso-scale turbulence in living fluids. Proc. Natl Acad. Sci. USA 109, 14308-14313 (2012).

40. Hernandez-Guzman, J. \& Weeks, E. R. The equilibrium intrinsic crystal-liquid interface of colloids. Proc. Natl Acad. Sci. USA 106, 15198-15202 (2009).

41. Gasser, U. et al. Real-space imaging of nucleation and growth in colloidal crystallization. Science 292, 258-262 (2001).

42. Aarts, D. G., Schmidt, M. \& Lekkerkerker, H. N. Direct visual observation of thermal capillary waves. Science 304, 847-850 (2004).

43. Patteson, A. E., Gopinath, A., Purohit, P. K. \& Arratia, P. E. Particle diffusion in active fluids is non-monotonic in size. Soft Matter 12, 2365-2372 (2016).

44. Chai, L., Vlamakis, H. \& Kolter, R. Extracellular signal regulation of cell differentiation in biofilms. Mrs. Bull. 36, 374-379 (2011).

45. Patteson, A. E., Gopinath, A., Goulian, M. \& Arratia, P. E. Running and tumbling with E. coli in polymeric solutions. Sci. Rep. 5, 15761 (2015).

46. Berg, H. C. \& Turner, L. Movement of microorganisms in viscous environments. Nature 278, 349-351 (1979).
47. Gloag, E. S. et al. Self-organization of bacterial biofilms is facilitated by extracellular DNA. Proc. Natl Acad. Sci. USA 110, 11541-11546 (2013)

48. Taylor, B. L. \& Koshland, D. E. Intrinsic and extrinsic light responses of Salmonella typhimurium and Escherichia coli. J. Bacteriol. 123, 557 (1975).

49. Conley, M. P. \& Berg, H. C. Chemical modification of streptococcus flagellar motors. J. Bacteriol. 158, 832 (1984).

50. Lu, S. et al. Loss of collective motion in swarming bacteria undergoing stress. Phys. Rev. Lett. 111, 208101 (2013).

\section{Acknowledgements}

We thank Edward Steager, Elizabeth Hunter, Somayeh Fahardi, Mark Goulian, Prashant Purohit, Julia Yeomans, and James Sethna for fruitful discussions. This work was supported by NSF-CBET-1437482. A.E.P was supported by an NSF Graduate Research Fellowship.

\section{Author contributions}

A.E.P. performed experiments. A.E.P. and A.G. designed and analyzed the experiments A.G. and A.E.P. formulated and analyzed the models for the interface dynamics. A.E.P., A.G., and P.E.A. wrote the manuscript.

\section{Additional information}

Supplementary Information accompanies this paper at https://doi.org/10.1038/s41467018-07781-y.

Competing interests: The authors declare no competing interests.

Reprints and permission information is available online at http://npg.nature.com/ reprintsandpermissions/

Publisher's note: Springer Nature remains neutral with regard to jurisdictional claims in published maps and institutional affiliations.

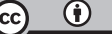

Open Access This article is licensed under a Creative Commons Attribution 4.0 International License, which permits use, sharing, adaptation, distribution and reproduction in any medium or format, as long as you give appropriate credit to the original author(s) and the source, provide a link to the Creative Commons license, and indicate if changes were made. The images or other third party material in this article are included in the article's Creative Commons license, unless indicated otherwise in a credit line to the material. If material is not included in the article's Creative Commons license and your intended use is not permitted by statutory regulation or exceeds the permitted use, you will need to obtain permission directly from the copyright holder. To view a copy of this license, visit http://creativecommons.org/ licenses/by/4.0/

(c) The Author(s) 2018 